\title{
Serum level of Total Antioxidant Status and Malondialdehyde in the Patients with Polycystic Ovarian Syndrome: a Comparative Study
}

\author{
Bibek Pun Magar, ${ }^{1}$ Dilli Bahadur Pun, ${ }^{2}$ Kapil Amgain, ${ }^{3}$ Keshavraj Joshi, ${ }^{4}$ Niranjan Satyal, ${ }^{5}$ Jharendra Bahadur Oli ${ }^{6}$
}

\section{Author Info:}

${ }^{1}$ Assistant Professor, Department of Biochemistry, ${ }^{2}$ Assistant Professor, Department of Physiology, ${ }^{3}$ Associate Professor, Department of Anatomy; Karnali Academy of Health Sciences, Jumla, Nepal

${ }^{4}$ Lecturer, Department of Biochemistry, Khaptad Medical Studies and Health Sciences, Council for Technical Education and Vocational Training (CTEVT)

${ }^{5}$ Consultant Biochemist, Department of Biochemistry, Chaurjahari Municipality Laboratory, West- Rukum, Nepal ${ }^{6}$ Pathology Technician,

Department of Laboratory, Royal Hobart Hospital, Australia

\section{*Corresponding Author:}

Mr. Bibek Pun Magar

Email:

bibekpunmagar9@gmail.com

\section{ABSTRACT}

Background: Polycystic ovary syndrome (PCOS) is a complex endocrine disorder in women of reproductive age and a major cause of infertility. Anti- Mullerian Hormone (AMH), a valid marker of ovarian function, is used for the diagnosis of PCOS. Oxidative stress (OS) occurs when harmful reactive oxygen species exceed the level of antioxidants. Malondialdehyde (MDA) is a marker of lipid peroxidation and increases in OS. The aim of this study is to assess the serum level of total antioxidant status (TAS) and malondialdehyde (MDA) in the premenopausal women with PCOS.

Methods: A cross-sectional analytical study was conducted at Padmashree Diagnostics, Department of Gynecology, India from April 2016 to March 2017. Census method was used to select the samples. The oxidative stress was measured by measuring the level of TAS and MDA. The data was recorded in structured proforma and and enter in SPSS version 16.0. The data were expressed in frequency, percentage, mean and standard deviation and the results obtained were analyzed using pearson's correlation and student's t-test. The $p$-value less than 0.05 was taken as statistically significant.

Results: The measured serum AMH level among the patients with PCOS was within normal range in $67.5 \%$ (27) and above normal range in $32.5 \%$ (13). The serum TAS level in normal AMH group was $814.0 \pm 291 \mu \mathrm{Mol} / \mathrm{L}$ and in above normal range group was720 $+240 \mu \mathrm{Mol} / \mathrm{L}$, which was statistically significant $(\mathrm{p}<0.001)$. Similarly, the serum level of MDA in normal AMH group was $6.64 \pm 0.21 \mu \mathrm{Mol} / \mathrm{L}$, and in above normal range group was $8.78 \pm 0.22 \mu \mathrm{Mol} / \mathrm{L}$, which is statistically significant $(\mathrm{p}=$ 0.01 ). The measured level of AMH was significantly associated with the increased level of MDA $(p<0.001)$ and decreased level of TAS $(p<0.001)$. The measured MDA level in high AMH group did show statistically significant than the normal AMH group. But it did not cross the normal reference limit, it underscores that the study subjects are in the process of initial phase of disease progression.

Conclusions: Serum level of TAS and MDA along with AMH are valuable diagnostic biomarker for the diagnosis of PCOS in the resource poor settings where ultrasound facility is unavailable. We recommend to conduct further research from different population taking larger sample to validate this finding and check for the sensitivity and specificity of the test.

Keywords: Antioxidants, Infertility, Oxidative biomarker, Oxidative stress, olycystic ovary disease

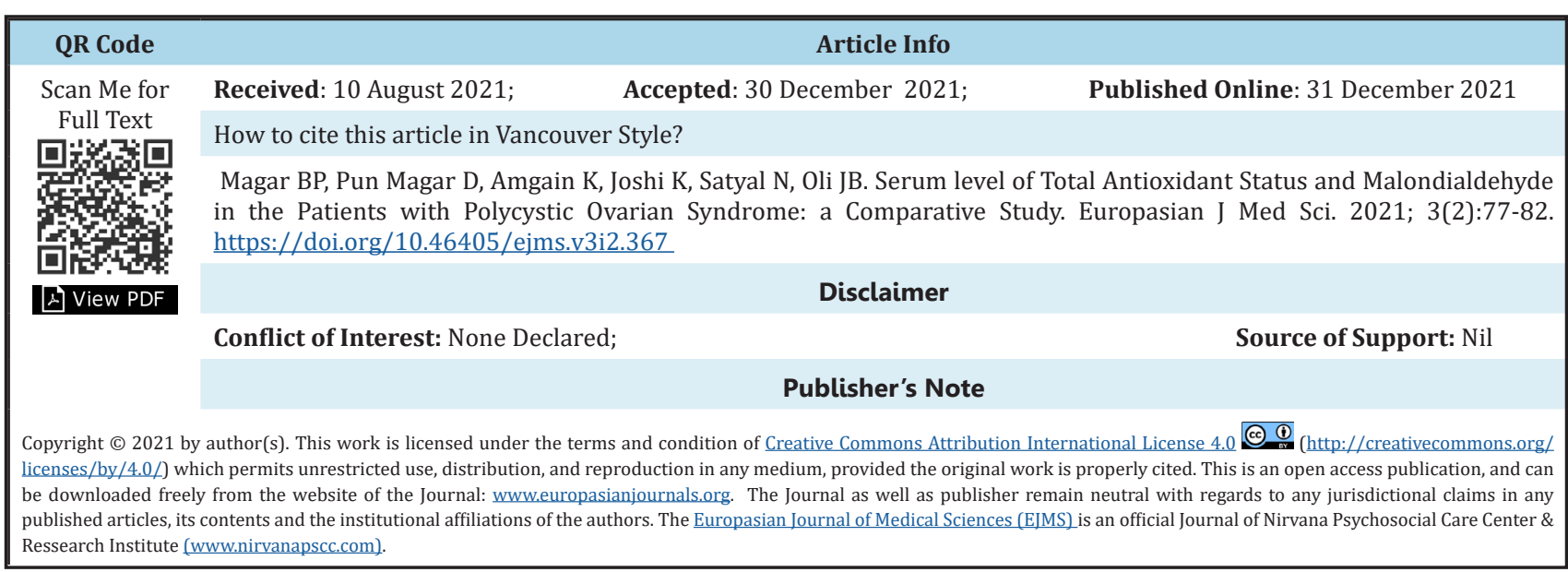




\section{INTRODUCTION}

Polycystic ovary syndrome (PCOS) is the most common endocrine disorder affecting $5-10 \%$ of female in their reproductive age ${ }^{1,2}$ It is the leading cause of ovulatory dysfunction causing female infertility worldwide., ${ }^{1,3}$ Researches showed that infertility in female is mainly hormonal. ${ }^{4}$ Ovary as an endocrine gland produces various hormones like estrogen, progesterone, antimullerian hormone (AMH) and little amount of androgen.

AMH is secreted by granulosa cells of the ovarian follicles, and is an important regulator of folliculogenesis in the ovaries. ${ }^{5}$ Some of the recent research has concluded that the serum levels of $\mathrm{AMH}$ is a valid indicator representing the overall ovarian function that determined the women's screening of fertility and diagnosis of PCOS and its level increases by 2 to 3 times in women with PCOS. ${ }^{6}$ However, the converse is not always valid. The women with high level of $\mathrm{AMH}$ who dont have polycystic ovaries are more fertile. ${ }^{7}$ The increase in the level of AMH has been involved in the pathogenesis of PCOS. Studies found that polycystic ovaries comprise a higher number of preantral and small antral follicles, indicating arrest of follicular development at the stage when $\mathrm{AMH}$ production is greatest. ${ }^{8,9}$

Oxidative stress (OS) is an imbalance in the systemic expression of reactive oxygen species (ROS) and the inability of the biological system to readily detoxify or repair the reactive intermediates, thus producing peroxides and free radicals that might cause damages to cell components likelipids, DNA and proteins. ${ }^{10-12}$ Various studies have measured antioxidant markers to correlate with OS and PCOS and the diverse clinical manifestations of metabolic syndrome including diabetes, obesity, and cardiovascular diseases. ${ }^{13}$ Malondialdehyde (MDA) is a marker of lipid peroxidation and increases in OS. MDA results from lipid peroxidation of polyunsaturated fatty acid. It is stable and can serve as a good biomarker. Increases ROS may cause intracellular damage causing an increase in MDA levels. ${ }^{5,14}$ Therefore, the role of OS in PCOS is becoming more important and new diagnostic biochemical markers are gaining much attention.

Although ultrasonography (USG) can confirm the presence of polycystic ovary in $90 \%$ of the case, it can't diagnose the PCOS due to the fact that polycystic ovary is the common feature of health women too. Therefore, the diagnosis of PCOS on the basis of hormonal assay is a promising method, however, analytic methods have to be standardized in order to establish the commonly accepted diagnostic norms. ${ }^{15}$ The aim of this study is to assess serum level of TAS and MDA, and correlate these biomarkers with the AMH levels in the premenopausal women.

\section{MATERIALS AND METHODS}

Study Design and Setting: A cross-sectional analytical study was conducted in Padmashree diagnostics, Department of Gynecology, Vijayanagar, Bengaluru, India from April 2016 to March 2017. The study was approved by Institutional Review Board, Padmashree Institute of Clinical Research, Bangalore.

Inclusion and Exclusion Criteria: Premenopausal women between the ages of 25 to 45 years with the clinical as well as radiological diagnosis of PCOD using Rotterdam criteria were included in the study. Two out of three Rotterdam criteria are required for the diagnosis of: Oligo/anovulation, hyperandrogenism (hirsutism, raised free testosterone), and radiological diagnosis of PCOS.

The participants with age below 25 and above 45 , those undergoing hormonal replacement therapy, late-onset congenital adrenal hyperplasia, and androgen secreted ovarian or adrenal tumors were excluded from the study.

Sampling Technique and Sample collection: Blood sample collection was done following aseptic laboratory procedure in gel clot activator vacuum tube. The census method was applied to identify the sample size. All patients meeting the inclusion criteria during the study period were enrolled in this study.

Ethics Statement: Both written as well as verbal consent was taken before blood sample collection from the participants. The study was approved by Institutional Review Board of Padmashree Institute of Clinical Research, Bangalore, India.

Procedure for Biochemical Tests: The blood specimens were received in the laboratory and centrifuged at $1800 \mathrm{xg} / 15 \mathrm{mins}$ to separate the cellular components and the cell-free serum processed for the analysis of routine biochemical parameters requested by the treating clinicians. The remaining specimens were aliquoted, labeled, and stored at $-20^{\circ} \mathrm{C}$ till further analysis. Aliquots of specimens, once thawed were used for the analysis on the same day and not be subjected to repeated freezing and thawing to avoid any pre-analytical errors. Three biochemical test parameters were studied; AMH, TAS and MDA.

\section{Analytical Methods:}

Serum AMH was estimated by Enzyme-linked fluorescence assay (ELFA) according to the manufacturer's instructions. ${ }^{16}$

Serum TAS was estimated by the modified Ferric reducing antioxidant power (FRAP) assay method. ${ }^{17}$

MDA level was estimated by a modified spectrophotometric method. ${ }^{6}$

Data analysis: Data were analyzed using the Statistical Package for the Social Sciences (SPSS version 16.0). All the values were expressed in frequency, percentage, mean and standard deviation. The student's t-test was applied for statistical comparison of two means and Pearson's correlation was applied to check the relationship between AMH, MDA and TAS level. The p-value less than 0.05 was considered as statistically significant. 


\section{RESULTS}

During the one year of the study period, a total of 1295 patients were visited in the OPD and 230 were with PCOS. Out these cases, 40 patients meet the inclusion criteria of the study and enrolled in this study. The prevalence of PCOS was $17.76 \%$. Out of the 40 patients, $67.5 \%(n=27)$ patients were with normal AMH level and $32.5 \%(n=13)$ were with high AMH level (Figure 1).

The mean and standard deviation of the AMH level in normal AMH group and high AMH group was $3.97 \pm 0.7$ and $6.73 \pm 1.98 \mathrm{ng} / \mathrm{mL}$ respectively which is statistically significant $(\mathrm{p}<0.001)$ (Table 1$)$.

The mean total antioxidants status (TAS) level was significantly lower among PCOS patients with normal high $\mathrm{AMH}$ as compared with high normal $\mathrm{AMH}$ $(\mathrm{p}<0.001)$. The mean value in the TAS in normal AMH group and high $\mathrm{AMH}$ group was $814.0 \pm 291 \mu \mathrm{Mol} / \mathrm{L}$ and $720 \pm 240 \mu \mathrm{Mol} / \mathrm{L}$ respectively (Table 2). Similarly, the level of MDA in normal AMH group and high $\mathrm{AMH}$ group was $6.64 \pm 0.21 \mu \mathrm{Mol} / \mathrm{L}$ and $8.78 \pm 0.22 \mu \mathrm{Mol} / \mathrm{L}$ respectively (Table 3).

$\mathrm{AMH}$ had strong positive correlation with MDA

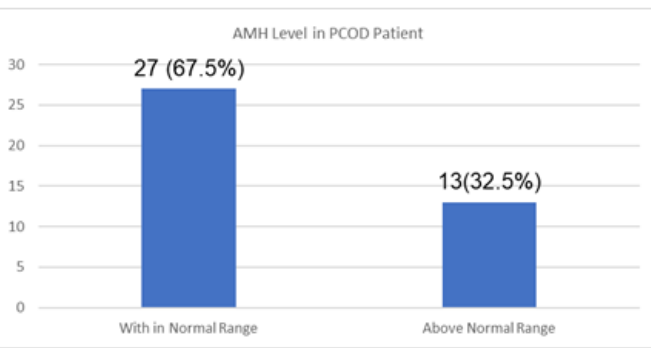

Figure 1: Frequency distribution of the Patient with PCOS with references to AMH level

Table 1: Serum level of AMH in both group of patients with PCOS

\begin{tabular}{lll}
\hline Category of PCOS Patients based on AMH Level & AMH Level (mean \pm SD) & p-value \\
\hline Normal AMH Group (n=27) & $3.97 \pm 0.7 \mathrm{ng} / \mathrm{mL}$ & $\mathrm{p}<0.001$ \\
High AMH Group (13) & $6.73 \pm 1.98 \mathrm{ng} / \mathrm{mL}$ & \\
\hline
\end{tabular}

Table 2: Serum level of TAS in both group of patients with PCOS

\begin{tabular}{lll}
\hline Category of PCOS Patients based on AMH Level & TAS level $((\operatorname{mean} \pm$ SD) & p-value \\
\hline Normal AMH Group $(\mathrm{n}=27)$ & $814.0 \pm 291 \mu \mathrm{Mol} / \mathrm{L}$ & $\mathrm{p}<0.001$ \\
High AMH Group (13) & $720 \pm 240 \mu \mathrm{Mol} / \mathrm{L}$ & \\
Normal Range of serum TAS level (reference value) & $1125 \pm 542 \mu \mathrm{Mol} / \mathrm{L}$ & \\
\hline
\end{tabular}

Table 3: Serum level of MDA in both group of patients with PCOS

\begin{tabular}{lll}
\hline Category of PCOS Patients based on AMH Level & MDA (mean \pm SD) & p-value \\
\hline Normal AMH Group ( $\mathrm{n}=27)$ & $6.64 \pm 0.21 \mu \mathrm{Mol} / \mathrm{L}$ & $\mathrm{P}<0.001$ \\
High AMH Group (13) & $8.78 \pm 0.22 \mu \mathrm{Mol} / \mathrm{L}$ & \\
Normal Range of serum MDA level (reference value) & $2.65 \pm 1.33 \mu \mathrm{Mol} / \mathrm{L}$ & \\
\hline
\end{tabular}

Table 4: Correlations between level of AMH, MDA and TAS

\begin{tabular}{lll}
\hline Serum Level of Biomarkers & $\mathbf{r}$-value & p-value \\
\hline AMH vs MDA & 0.83 & $<0.001$ \\
AMH vs TAS & -0.36 & 0.02 \\
TAS vs MDA & -0.72 & $<0.001$ \\
\hline
\end{tabular}
$(p=0.02)$ which was statistically significant. Similarly TAS had strong but negative correlation with MDA. (Table 4)

\section{DISCUSSION}

PCOS is a multi-factorial disease having complex hormonal disturbances and is a major cause of infertility in female. The present study was undertaken to analyze the OS in females having PCOS. We correlated OS by measuring serum TAS and MDA in a total of 40 participants having PCOS. Among them, $67.5 \%(n=27)$ had normal AMH level and 32.5\% $(n=13)$ had high AMH level. The findings of the study showed the AMH level was statistically significant against reference interval which showed that these premenopausal women with high $\mathrm{AMH}$ level group were suffering from PCOS which was similar to the results of other studies done by Kohzadi et al. ${ }^{6}$ and Woo et al. ${ }^{17}$

Various hormones have play vital role in maintaining the ovarian function and many of them are changed during the disease of ovary like PCOS. AMH is a hormone produced primarily by granulocytes of preantral follicles of ovaries in woman of reproductive life. Its secretion reasonably maintains the steady state in menstrual cycle, with the number of follicles and ovarian reserve. Researches has showed that AMH level can act as novel marker of ovulatory dysfunction. As 
AMH level can be used as marker of ovarian reserve and viability to ovulate. Thus, these results demonstrated that the AMH level is strongly linked with ovarian antral counts. These factors make AMH level an ideal marker for ovarian reserve. However, with the help of serum AMH level only, the diagnosis of PCOS cannot be made in the patients suspected with PCOS. A previous study found that AMH level is associated with severity of PCOS. ${ }^{18}$ Other studies found that increased level of $\mathrm{AMH}$ in the female without sign/symptoms of PCOS had significantly higher fertility. ${ }^{7}$ So the AHM level only is unable to diagnose the PCOS.

Our study showed that the level of TAS decreases in the patient diagnosed with PCOS. Moreover, the result showed that the level of TAS was decrease significantly in the PCOS patient with high AMH group than in normal $\mathrm{AMH}$ group. In the present study, the total antioxidant status among pre-menopausal woman of normal and high AMH level subjects had $814.0 \pm 291 \mu \mathrm{Mol} / \mathrm{L}$ and $720 \pm 240 \mu \mathrm{Mol} / \mathrm{L}$ respectively which was significantly lower than the reference interval in both the normal and high $\mathrm{AMH}$ groups. These findings showed that both groups were under oxidative stress as compared to that of normal reference intervals. These findings were similar to that of the previous study done by Fenkci et al, Maleedhu et al, and Fathi et al. ${ }^{19-21}$ However, our study result was contrary to Verit et $\mathrm{al}^{22}$ in which TAS was significantly higher in PCOS participants. The observed finding of the present study reveals that the normal and high AMH groups were under oxidative stress compare to that of normal reference interval. On the contrary, the TAS decreases showed the presence of undue disturbance in redox homeostasis in PCOS. This result was in agreement with Shirsath et al. ${ }^{23}$ where they reported a significant increase in the mean of MDA ( $p>0.001)$ in PCOS women than that of controls group.

The reasons behind the reduced level of TAS is due to increase in level of free radicals produced from the redox reactions that can reduce from proteins, lipids as well as DNA damage and thereby neutralize, reactive oxygen species such as hydrogen peroxide etc. ${ }^{24}$ If the defense mechanism cannot overcome the redox reactions would eventually happens to damage the body tissue it is like aging process. The total antioxidant status among premenopausal women of normal and high AMH level group was found lower than the reference interval. ${ }^{19-21}$

Similarly, present study found that the level of MDA was significantly higher in the PCOS patients. The observed level of MDA is significantly increased in the PCOS patient with high AMH group than normal AMH group $(8.78 \pm 0.22 \mu \mathrm{Mol} / \mathrm{L}$ and $6.64 \pm 0.22 \mu \mathrm{Mol} / \mathrm{L})$ than that of reference level $(2.65 \pm 1.33 \mu \mathrm{Mol} / \mathrm{L})$ respectively which was similar to the results of other studies ${ }^{25-27}$ but not in agreement with Dursun et al..$^{28}$ and Karadeniz et al. ${ }^{29}$ which reported no statistical variation regarding the mean of MDA level between PCOS and the control group. The MDA level helps in evaluating lipid peroxidation as a consequence of oxidative stress.
Therefore, increased serum MDA concentrations might be a result of increased generation of ROS due to the generation of excessive oxidative damage in these patients. These oxygen species in turn can oxidize many other important biomolecules including membrane lipids. Though the exact mechanism has not been clearly understood, hyperandrogenemia in PCOS may be the reason for these higher MDA levels. ${ }^{18}$ In present study $\mathrm{AMH}$ exhibited strong correlation with MDA $(\mathrm{r}=+0.83$, $\mathrm{p}<0.001)$. In this study, the mean serum MDA level was significantly increased in the high $\mathrm{AMH}$ group than the normal AMH group. But found lower than the reference interval. This suggested that an increase in lipid content oxidative biomarker MDA in the high AMH group than the low AMH group.

TAS showed statistically significant negative correlation with MDA ( $\mathrm{r}=-0.72, \mathrm{p}<0.001)$. The increased level of MDA and decrease level of TAS in serum showed that there is increased amount of lipid peroxidation as a consequence of oxidative stress in the patients with PCOS. ${ }^{24}$ Thus, the measure of TAS as well as MDA in body fluids is functionally important in handling oxidative stress in PCOD. Hence, in PCOS patients, the antioxidants status can be of help to fight against these free radicals and prevent future complications. So, the present study was an attempt to further the routine chemistry parameters along with special test parameters like $\mathrm{AMH}$ to analyze and relate the findings with the clinical diagnosis for differential diagnostic significance in PCOS. AMH showed statistically significant but weak negative correlation with TAS $(\mathrm{r}=-0.36, \mathrm{p}=0.02)$.

The major limitations of this study being a single centered study with small sample size. Also, other variables/parameters and markers were not assessed in the study. To understand the novelty of interrelation in maintaining the redox homeostasis there is urgent need to call for a larger population based prospective study over a period of time along with the monitoring of the therapeutic outcome is likely to validate the prognostic significance if any, of oxidative biomarker in polycystic ovarian disease.

\section{CONCLUSION}

This study confirms that the low-level of TAS and highlevel of MDA served as a diagnostic biomarker for PCOS in the patients with high level of $\mathrm{AMH}$ in Indian sub-population. It is valuable to the clinician working in resource poor settings for the diagnosis of PCOS by assessing the lever of AMH, TAS and MDA alone which is more convenient and less expensive in the clinical setting where ultrasonological diagnosis is unavailable. We recommend to conduct further research in this area taking large sample from different population to validate these findings and check for the sensitivity and specificity of the test.

Acknowledgements: We would like to acknowledge Dr. Suresh Babu SV for his unconditional support and guidance throughout the study period. We would 
like to thank all the patients who give us consent to participate in our study.

\section{REFERENCES}

1. Ndefo UA, Eaton A, Green MR. Polycystic ovary syndrome: a review of treatment options with a focus on pharmacological approaches. Pharmacy and therapeutics. 2013;38(6):336-55. [ $\underline{\text { PubMed | Full }}$ Text | Google Scholar]

2. Zuo T, Zhu M, Xu W. Roles of oxidative stress in polycystic ovary syndrome and cancers. Oxid Med Cell Longev. 2016;2016: 8589318. [PubMed | Full Text | DOI | Google Scholar]

3. March WA, Moore VM, Willson KJ, Phillips DIW, Norman RJ, Davies MJ. The prevalence of polycystic ovary syndrome in a community sample assessed under contrasting diagnostic criteria. Hum Reprod. 2010;25(2):544-51. [PubMed | Full Text | DOI | GoogleScholar]

4. Luciano AA, Lanzone A, Goverde AJ. Management of female infertility from hormonal causes. Int J Gynaecol Obstet. 2013 Dec;123:S9-17. [Full Text | GoogleScholar | DOI]

5. Knight JA, Smith SE, Kinder VE, Anstall HB. Reference intervals for plasma lipoperoxides: age-, sex-, and specimen-related variations. Clin Chem. 1987;33(12):2289-91. [PubMed | GoogleScholar]

6. Kohzadi M, Khazaei MR, Choobsaz F, Khazaei M. Relationship between serum levels of anti-mullerian hormone, adiponectin and oxidative stress markers in patients with polycystic ovary syndrome. Int J Fertil Steril. 2020 Apr;14(1):27-33. doi: 10.22074/ ijfs.2020.5809. [PubMed | DOI | Full Text]

7. Yue CY, Lu LK, Li M, Zhang QL, Ying CM. Threshold value of anti-Mullerian hormone for the diagnosis of polycystic ovary syndrome in Chinese women. PLoS One. 2018 Aug 28;13(8):e0203129. [PubMed | GoogleScholar | Full Text]

8. Pigny P, Merlen E, Robert Y, Cortet-Rudelli C, Decanter C, Jonard S, et al. Elevated Serum Level of Anti-Mullerian Hormone in Patients with Polycystic Ovary Syndrome: Relationship to the Ovarian Follicle Excess and to the Follicular Arrest. J Clin Endocrinol Metab. 2003;88(12):5957-62. [PubMed | Full Text | DOI | GoogleScholar]

9. Laven JSE, Mulders AGMGJ, Visser JA, Themmen $\mathrm{AP}$, De Jong FH, Fauser BCJM. Anti-Müllerian HormoneSerum Concentrations in Normoovulatory and Anovulatory Women of Reproductive Age. J Clin Endocrinol Metab. 2004;89(1):318-23. [PubMed | Full Text | DOI | GoogleScholar]

10. Giacco F, Brownlee M. Oxidative stress and diabetic complications. Circ Res. 2010;107(9):1058-70. [PubMed | Full Text | GoogleScholar]

11. Agarwal A, Gupta S, Sharma RK. Role of oxidative stress in female reproduction. Reprod Biol Endocrinol. 2005;3:1-21. [ [PubMed | Full Text | DOI | GoogleScholar]

12. Sabuncu T, Vural H, Harma M, Harma $M$. Oxidative stress in polycystic ovary syndrome and its contribution to the risk of cardiovascular disease. Clin Biochem. 2001;34(5):407-13. [PubMed | Full Text | DOI | GoogleScholar]

13. Lee JY, Baw C-K, Gupta S, Aziz N, Agarwal A. Roles of Oxidative Stress in Policystic Ovary Syndrome. Serbian J Exp Clin Res. 2019;6(2):96-107. [ [Full Text]

14. Abuja PM, Albertini R. Methods for monitoring oxidative stress, lipid peroxidation and oxidation resistance of lipoproteins. Clin Chim Acta. 2001;306(1-2):1-17. [ubMed | Full Text | DOI | GoogleScholar]

15. Bachanek M, Abdalla N, Cendrowski K, Sawicki W. Value of ultrasonography in the diagnosis of polycystic ovary syndrome-literature review. J Ultrason. 2015 Dec;15(63):410-22. [PubMed | GoogleScholar | Full Text | DOI]

16. Sevgi R, Erdem H, Karaşahin T, et al. Determination of the relationship between serum anti-Müllerian hormone level and superovulatory response in Simmental cows. Reprod in Domest Anim. 2019 Oct;54(10):1322-9. [라Med | GoogleScholar | Full

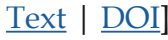

17. Woo HY, Kim KH, Rhee EJ, Park H, Lee MK. Differences of the association of anti-Müllerian hormone with clinical or biochemical characteristics between women with and without polycystic ovary syndrome. Endocr J. 2012;59(9):781-9. [PubMed | Full Text | DOI | GoogleScholar]

18. Tal R, Seifer CM, Khanimov M, Seifer DB, Tal O. High serum Antimullerian hormone levels are associated with lower live birth rates in women with polycystic ovarian syndrome undergoing assisted reproductive technology. Reprod Biol Endocrinol. 2020 Dec;18(1):1-8. [PubMed | GoogleScholar | Full Text | DOI]

19. Fenkci V, Fenkci S, Yilmazer $M$, Serteser $M$. Decreased total antioxidant status and increased oxidative stress in women with polycystic ovary syndrome may contribute to the risk of cardiovascular disease. Fertil Steril. 2003;80(1):1237. [PubMed | Full Text | DOI | GoogleScholar]

20. Maleedhu P, Rao P P, Kodumuri PK. Antioxidant Status in Women with Polycystic Ovary Syndrome. Res Rev J Med Heal Sci. 2014;3(Supplement 2):91-6. [PubMed | Full Text | DOI]

21. Fathi F. Biomarkers of Oxidative Stress in Polycystic Ovary Disorder. Ann Coll Med Mosul. 2020;41(2):112-6. [Full Text]

22. Verit FF, ErelO. Oxidative stress in nonobese women with polycystic ovary syndrome: Correlations with 
endocrine and screening parameters. Gynecol Obstet Invest. 2008;65(4):233-9. [ㅁubMed | Full Text | DOI | GoogleScholar]

23. Shirsath A, Aundhakar N, Kamble P. Study of oxidative stress and antioxidant levels in polycystic ovarian disease. Int $\mathrm{J}$ Healthc Biomed Res. 2015;3(04):16-24. [GoogleScholar | FullText]

24. Mohammadi M. Oxidative stress and polycystic ovary syndrome: a brief review. Int J Prev Med. 2019;10:86. [PubMed | GoogleScholar | Full Text | DOI]

25. Zhang D, Luo WY, Liao H, Wang CF, Sun Y. The effects of oxidative stress to PCOS. Sichuan Da Xue Xue Bao Yi Xue Ban. 2008 May;39(3):421-3. [PubMed [GoogleScholar]

26. Kuşçu NK, Var A. Oxidative stress but not endothelial dysfunction exists in non-obese, young group of patients with polycystic ovary syndrome. Acta Obstet Gynecol Scand. 2009 Jan 1;88(5):612-7. [PubMed | Full Text | DOI | GoogleScholar]

27. Oyebanji O, Asaolu M. Assessment of antioxidant status of women with polycystic ovarian syndrome. Asian Pacific J Reprod [Internet]. 2020;9(1):9-15. [Full Text | DOI | GoogleScholar]

28. Dursun P, Demirtaş E, Bayrak A, Yarali H. Decreased serum paraoxonase 1 (PON1) activity: an additional risk factor for atherosclerotic heart disease in patients with PCOS?. Hum Reprod. 2006 Jan 1;21(1):104-8. [GoogleScholar | Full Text]

29. Karadeniz M, Erdoğan M, Tamsel S, Zengi A, Alper $\mathrm{GE}$, Çağlayan $\mathrm{O}$, et al. Oxidative stress markers in young patients with polycystic ovary syndrome, the relationship between insulin resistances. Exp Clin Endocrinol Diabetes. 2008;116(4):231-5. [ [PubMed | Full Text | GoogleScholar | DOI] 